\title{
Alginate Microcapsule Technology and Impacts on Cell Therapy Development
}

\author{
Marwa Belhaj ${ }^{1}$, Vinal Menon ${ }^{1}$, Bärbel Rohrer ${ }^{2,3}$ and, Jay Potts ${ }^{1,4}$ \\ ${ }^{1 .}$ Department of Cell Biology and Anatomy, School of Medicine, University of South Carolina, \\ Columbia, USA. \\ 2. Department of Ophthalmology, Medical University of South Carolina, Charleston, South \\ Carolina, USA. \\ ${ }^{3 .}$ Ralph H. Johnson VA Medical Center, Charleston, South Carolina, USA. \\ 4. Biomedical Engineering Program, College of Engineering and Computing, University of South \\ Carolina, Columbia, USA.
}

Cell-based therapies represent a revolutionary bio-technique that has been applied widely in medicine. Recently, they have been successfully applied in treatment of neurodegenerative diseases, eye diseases, cardiovascular disease, diabetes, liver disease and cancer. Suppression of the host immune system is considered the main challenge in such therapies. In order to avoid the side effects of immunosuppressive drugs, encapsulating cells into polymeric matrices is considered a promising strategy. Encapsulation systems utilize permeable materials that allow diffusion of nutrients, waste and therapeutic factors into and out of cells, while masking the cells from the host immune response.

Alginate is one of the widely used polymers in microencapsulation due to its biocompatibility and costeffective production. It is a natural polysaccharide derived from brown seaweed and has the ability to polymerize rapidly in the presence of cations to form a porous matrix. Electrospray method, as a microencapsulation technique, has been previously used to encapsulate peptides and proteins successfully using alginate [1]. According to this method, the basic polymer is placed in a positively charged syringe attached to a high voltage generator. A syringe pump is used to control the flow rate of the polymer, which is extruded into a grounded solution $(0.15 \mathrm{mM}$ calcium chloride). A second coating of poly-l-ornithine (PLO) polymer can be used to increase the integrity of alginate microcapsules.

Long-term, ocular drug delivery is a big challenge for the development of treatments in chronic retinal diseases such as retinitis pigmentosa (RP), age-related macular degeneration (AMD) of glaucoma. Different techniques for delivery include ocular inserts, biodegradable polymeric systems, or collagen shields; however, those still do not address the issue of long-term availability of a drug, especially biologics. ARPE-19, a spontaneously arising human RPE cell line, has been used in long-term cell therapy experiments due to its lifetime functionality. These cells can be transfected to express the desired biologics such as growth factors, blocking antibodies or other designer drugs for extended periods of time. In clinical trials for RP and dry AMD/ geographic atrophy (GA), encapsulating these cells in a semipermeable polymer outer membrane, sealed with a medical-grade sealant, and an internal polyethylene terephthalate yarn scaffold that supports the cells, long-term production of a growth factor without system exposure, no antibodies generated to encapsulated cells or their products, was demonstrated [2]. Here we hypothesize that alginate encapsulation technique using high voltage method preserves the metabolic activity of genetically engineered ARPE-19 cells resulting in effective and safe long-term drug delivery for treatment of retinal degeneration.

The present study shows the successful formation of alginate-poly-L-ornithine microcapsules using the above-mentioned method, according to specific parameters, to produce microcapsules about $200 \mu \mathrm{m}$ in 
diameter (Figure 1). Importantly, these microcapsules were successfully freeze-dried (lyophilized) in order to increase their shelf-life storage, which would be beneficial for their clinical use; as it has been shown previously in lyophilization of plasma, proteins, and cells [3]. In addition, we were able to show that freeze-dried microcapsules regained their spherical morphology after reconstitution in deionized water (Figure 1). By applying this system for encapsulating ARPE-19 cells, we have demonstrated a successful encapsulation of this type of cells that showed their ability to survive the applied high voltage power during encapsulation process. Cells were able to preserve their viability after encapsulation and they were cultured in media at $37^{\circ} \mathrm{C} / \mathrm{Co}_{2}$ (Figure $2 \mathrm{~B}, 2 \mathrm{C}$ ). Cells showed viability in culture for up to two months after encapsulation. To evaluate the proliferation capacity of encapsulated cells, they were released from the microcapsules after dissolving sodium alginate structure. Released cells were cultured in media at $37^{\circ} \mathrm{C} / \mathrm{Co}_{2}$, and showed a proliferative ability with the same rate as controls. Through the results of this work, we have showed that high voltage encapsulation can be utilized to develop cellbased therapies with alginate microcapsules. Also, we have demonstrated that freeze-dried microcapsules retain the integrity of their structure after reconstitution; therefore, this approach can be further enhanced for the benefit of the cell therapy development.

\section{References:}

[1] Moore K et al, Microsc Microanal (2013) p. 213-226.

[2] Kauper K et al, Invest Ophthalmol Vis Sci (2012) p. 7484-7491.

[3] Wikström J et al, Eur J Pharm Sci (2012) p. 520-526.

[4] The authors acknowledge funding from Cook Biotech, FirstString Research Inc, NIH 2 P20RR016434-06

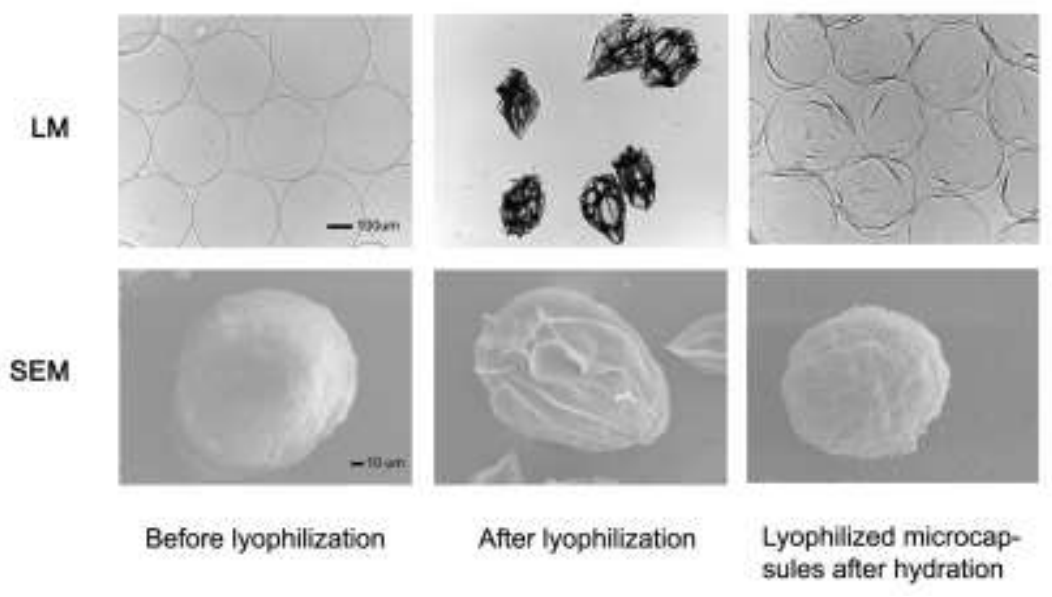

Figure 1. Light microscope and SEM images of empty alginate microcapsules before and after lyophilization. Scale bars are $100 \mu \mathrm{m}(\mathrm{LM})$ and $10 \mu \mathrm{m}(\mathrm{SEM})$, respectively.
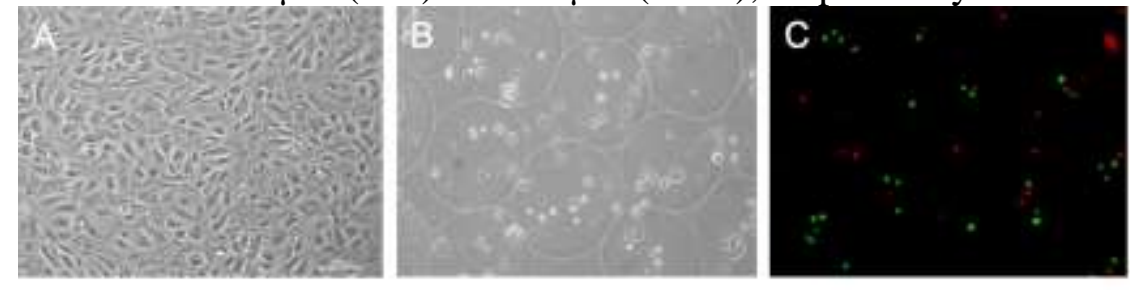

Figure 2. (a) Cultured ARPE-19 cells in media. (b) Alginate encapsulated ARPE-19 cells after 1 hour of encapsulation. (c) Live-dead staining with Calcein AM (Green/live) and Ethidium Homodimer (Red/Dead). Scale bars are $100 \mu \mathrm{m}$. 